\title{
STUDY ON CLINICAL PROFILE AND LABORATORY PARAMETERS OF ENTERIC FEVER CASES WITH SPECIAL REFERENCE TO ANTIBIOTIC SENSITIVITY PATTERN
}

\author{
Somnath Maity1, Suparna Guha²
}

1Postgraduate Trainee, Department of Paediatric Medicine, Vivekananda Institute of Medical Sciences, Kolkata, West Bengal, India. ${ }^{2}$ Associate Professor, Department of Paediatric Medicine, Vivekananda Institute of Medical Sciences, Kolkata, West Bengal, India.

\begin{abstract}
BACKGROUND
Enteric fever is a serious infection caused by salmonella typhi and paratyphi. It is endemic in low and middle socio-economic conditions. It is transmitted mainly by feco-oral route. With the passage of time, the disease spectrum has undergone a sea of changes. Nowadays multidrug resistant infections are often encountered. These are very difficult to treat. In this study, we aimed to investigate the clinical features and laboratory features with special reference to the antibiotic sensitivity pattern. We undertook this study to identify the changing clinical scenarios, the laboratory parameters and above all the antibiotic sensitivity patterns which can guide us to initiate judicious treatment.
\end{abstract}

ABSTRACT

\section{METHODS}

90 clinically suspected cases of enteric fever were initially selected. Of these, 50 patients whose blood culture was positive for salmonella typhi infection, were selected. Their clinical features, laboratory findings and antibiotic sensitivity pattern were studied in detail. All the data collected were then analysed using descriptive statistics and Chi square analysis.

\section{RESULTS}

Fever was universally present along with hepatosplenomegaly. Majority of the patients responded to intravenous ceftriaxone. 90 case records of clinically suspected cases of enteric fever were initially selected, out of which 40 cases were discarded as blood culture was not done or turned out to be negative. Remaining 50 cases were blood culture positive for salmonella typhi. These 50 cases were selected, their data collected and analysed.

\section{CONCLUSIONS}

Enteric fever is a common cause of fever in developing countries. Early diagnosis and treatment with proper selection of antibiotics is necessary to avoid major complication, later on. In spite of this, ceftriaxone still remains the drug of choice in uncomplicated enteric fever. Oral Azithromycin and Cefixime also showed good efficacy and a high sensitivity pattern was noted with both of them.

\section{KEY WORDS}

Blood Culture, Hepatosplenomegaly

HOW TO CITE THIS ARTICLE: Maity S, Guha S. Study on clinical profile and laboratory parameters of enteric fever cases with special reference to antibiotic sensitivity pattern. J. Evolution Med. Dent. Sci. 2019;8(25):2007-2011, D0I: $10.14260 /$ jemds/2019/441

\section{BACKGROUND}

Enteric fever caused by salmonella typhi and paratyphi is not very uncommon in the developing countries. With the passage of time, the disease spectrum has undergone a sea of changes. Nowadays multidrug resistant infections are often encountered. These are very difficult to treat. We undertook this study to identify the changing clinical scenarios, the laboratory parameters and above all the antibiotic sensitivity pattern which can guide us to judicious treatment.

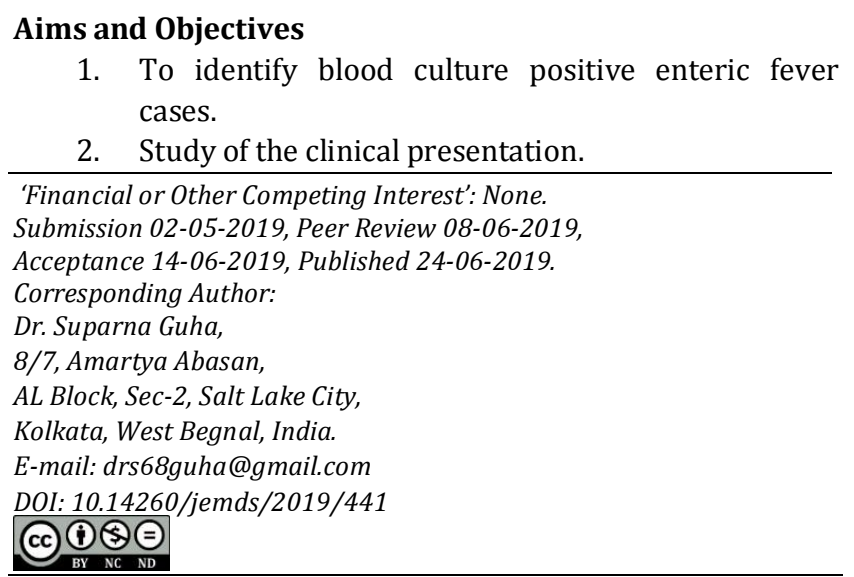

3. Evaluate the haematological, biochemical and radiological parameters.

4. Study the antibiotic sensitivity pattern of the identified salmonella organism.

\section{METHODS}

Type of Study

Retrospective cross-sectional hospital based observational study.

\section{Place of Study}

Inpatient Paediatric Medicine department of Vivekananda Institute of Medical Sciences

Period of Study

January 2017 to June 2018.

\section{Number of Subjects}

50 blood culture positive enteric fever cases.

\section{Inclusion Criteria}

Febrile children $<12$ years of age diagnosed as enteric fever by blood culture, have been included in the study. 


\section{RESULTS}

90 case records of clinically suspected cases of enteric fever were initially selected, out of which 40 cases were discarded as blood culture was not done or turned out to be negative. Remaining 50 cases were blood culture positive for salmonella typhi. These 50 cases were selected, their data collected and analysed.

\begin{tabular}{|c|c|c|}
\hline Positive Blood Culture & No. of Cases (n=50) & Percentage (5) \\
\hline S. typhi & 50 & $100 \%$ \\
\hline S. paratyphi & 0 & $0 \%$ \\
\hline Table 1. The Above Table Shows That All Blood Culture Positive \\
Cases Showed Growth of Salmonella typhi \\
\hline
\end{tabular}

\begin{tabular}{|c|c|c|}
\hline Age in Years & No. of Cases (n=50) & Percentage (\%) \\
\hline $0-5$ & 20 & 40 \\
\hline $6-12$ & 30 & 60 \\
\hline Total & $\mathbf{5 0}$ & $\mathbf{1 0 0}$ \\
\hline \multicolumn{3}{|c|}{ Table 2. Age Distribution } \\
\hline
\end{tabular}

\begin{tabular}{|c|c|c|}
\hline Sex & No. of Cases (n=50) & Percentage \% \\
\hline Male & 26 & 52 \\
\hline Female & 24 & 48 \\
\hline Total & $\mathbf{5 0}$ & $\mathbf{1 0 0}$ \\
\hline \multicolumn{3}{|c|}{ Table 3. Sex Distribution } \\
\hline $\begin{array}{l}\text { The above data shows that there was no significant sex difference in our } \\
\text { study. }\end{array}$ \\
\hline
\end{tabular}

\begin{tabular}{|c|c|c|c|}
\hline Age in Yrs. (n=50) & Male & Female & Total \\
\hline $0-5$ & 14 & 6 & 20 \\
\hline $6-12$ & 12 & 18 & 30 \\
\hline Total & $\mathbf{2 6}$ & $\mathbf{2 4}$ & $\mathbf{5 0}$ \\
\hline \multicolumn{3}{|c|}{ Table 4. Age with Sex Distribution } \\
\hline $\begin{array}{l}\text { Out of the } 50 \text { blood-culture positive cases, among 0-5 years age group, } \\
\text { majority were males (70\%) and among 6-12 years age group majority } \\
\text { were females (60\%). }\end{array}$ \\
\hline
\end{tabular}

\begin{tabular}{|c|c|c|}
\hline Duration of Fever (Days) & No. of Cases (n=50) & Percentage (\%) \\
\hline $1-6$ & 0 & 0 \\
\hline $7-14$ & 47 & 94 \\
\hline$>14$ & 3 & 6 \\
\hline Total & $\mathbf{5 0}$ & $\mathbf{1 0 0}$ \\
\hline \multicolumn{2}{|c|}{ Table 5. Duration of Fever at The Time of Admission } \\
\hline
\end{tabular}

\begin{tabular}{|c|c|c|c|}
\hline Serial No. & Symptom & 0-5 yrs. (n=20) & $\mathbf{6 - 1 2}(\mathbf{n}=\mathbf{3 0})$ \\
\hline 1. & Fever & $20(100 \%)$ & $30(100 \%)$ \\
\hline 2. & Chills & $3(15 \%)$ & $2(6.6 \%)$ \\
\hline 3. & Headache & 0 & $2(6.6 \%)$ \\
\hline 4. & Vomiting & $12(60 \%)$ & $13(43.3 \%)$ \\
\hline 5. & Pain abdomen & $13(65 \%)$ & $20(66.6 \%)$ \\
\hline 6. & Constipation & $14(70 \%)$ & $19(63.3 \%)$ \\
\hline 7. & Diarrhoea & $3(15 \%)$ & $3(10 \%)$ \\
\hline 8. & Skin rash & $1(5 \%)$ & $1(3.3 \%)$ \\
\hline 9. & Arthralgia & 0 & 0 \\
\hline 10. & Urinary symptom. & 0 & $2(6.6 \%)$ \\
\hline 11. & Cough & 0 & 0 \\
\hline 12. & GI Bleed & 0 & 0 \\
\hline \multicolumn{4}{|c|}{ Table 6. Clinical Symptoms in Different Ages } \\
\hline
\end{tabular}

\begin{tabular}{|c|c|c|c|}
\hline Sl. No. & Signs & 0-5 Yrs. (n=20) & 6-12 Yrs. (n=30) \\
\hline 1. & Pyrexia & $20(100 \%)$ & $30(100 \%)$ \\
\hline 2. & Coated tongue & $20(100 \%)$ & $28(93.3 \%)$ \\
\hline 3. & Rash, macula papular & $1(5 \%)$ & $1(3.33 \%)$ \\
\hline 4. & Relative Bradycardia & $3(15 \%)$ & $11(36.6 \%)$ \\
\hline 5. & Pallor & $10(50 \%)$ & $14(46.6 \%)$ \\
\hline 6. & Jaundice & $2(10 \%)$ & 0 \\
\hline 7. & Hepatosplenomegaly & $20(100 \%)$ & $30(100 \%)$ \\
\hline 8. & Complications & $3(15 \%)$ & $6(20 \%)$ \\
\hline \multicolumn{4}{|c|}{ Table 7. Clinical Symptoms in Different Age Groups } \\
\hline
\end{tabular}

\begin{tabular}{|c|c|c|}
\hline Widal Reaction & No. of Cases (n=50) & Percentage (\%) \\
\hline Positive & 26 & 52 \\
\hline Negative & 24 & 48 \\
\hline Table 8. Widal Test Result in Blood C/S Positive Patients (In all \\
Cases Test Done After $>$ /= 7 Days of Starting of Fever) \\
\hline
\end{tabular}

\begin{tabular}{|c|c|c|c|c|}
\hline & & Blood Culture + & Blood Culture - & Total \\
\hline Widal & Positive & 26 & 9 & 35 \\
\hline & Negative & 24 & 31 & 55 \\
\hline Total & & $\mathbf{5 0}$ & $\mathbf{4 0}$ & $\mathbf{9 0}$ \\
\hline \multicolumn{6}{|c|}{ Table 9. Comparison of Widal Test with Blood Culture } \\
\hline
\end{tabular}

\begin{tabular}{|c|c|c|}
\hline Antibiotic & No. of Cases (n=50) & Percentage (\%) \\
\hline Co-amoxiclav & 45 & 90 \\
\hline Cefixime & 47 & 94 \\
\hline Azithromycin & 48 & 96 \\
\hline Ofloxacin & 4 & 8 \\
\hline Ceftriaxone & 48 & 96 \\
\hline Cefepime & 48 & 96 \\
\hline Meropenem & 49 & 98 \\
\hline Aminoglycosides & 2 & 4 \\
\hline Nalidixic Acid & 0 & 0 \\
\hline \multicolumn{2}{|c|}{ Table 10. Antibiotic Sensitivity Pattern } \\
\hline
\end{tabular}

\begin{tabular}{|c|c|c|c|c|c|c|}
\hline 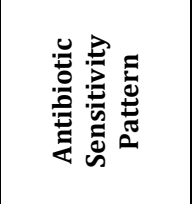 & 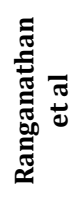 & 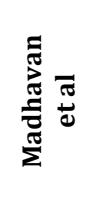 & 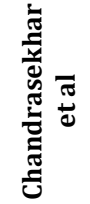 & 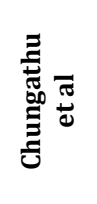 & 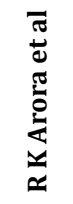 & 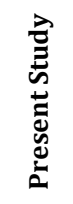 \\
\hline Co-amoxiclav & $70 \%$ & $88.2 \%$ & $87 \%$ & $96 \%$ & $88 \%$ & $90 \%$ \\
\hline Cefepime & $100 \%$ & $90 \%$ & $92 \%$ & $96 \%$ & $92 \%$ & $94 \%$ \\
\hline Azithromycin & $60 \%$ & $86 \%$ & $84 \%$ & $88 \%$ & $90 \%$ & $96 \%$ \\
\hline Quinolones & $87 \%$ & $2.6 \%$ & $97 \%$ & $94 \%$ & $90 \%$ & $8 \%$ \\
\hline Ceftriaxone & $100 \%$ & $90 \%$ & $87.5 \%$ & $100 \%$ & $92 \%$ & $96 \%$ \\
\hline Meropenem & $100 \%$ & $98 \%$ & $100 \%$ & $96 \%$ & $96 \%$ & $98 \%$ \\
\hline Aminoglycosides & $20 \%$ & $2.6 \%$ & $100 \%$ & $10 \%$ & $16 \%$ & $4 \%$ \\
\hline \multicolumn{7}{|c|}{$\begin{array}{c}\text { Table 11. Comparison of Antibiotic Sensitivity Pattern with Other } \\
\text { Studies }\end{array}$} \\
\hline
\end{tabular}

\section{Exclusion Criteria}

Febrile children who were clinically suspected to be enteric fever, but blood culture negative was not included in the study.

The case sheets of these patients were collected from the record section of the hospital.

Several data including clinical features, culture sensitivity pattern, laboratory parameters and antibiogram data were retrieved from the hospital records.

Blood culture was performed by BACTEC method, collecting $5 \mathrm{cc}$ venous blood and diluting it 4 times under normal circumstances and 10 times if treated with antibiotics and then injecting into a bulb containing $20-30 \mathrm{cc}$ of $5 \%$ bile broth. The bulb was incubated for 24-48 hours at 37 degree centigrade and subcultures were made on McCaskey's medium.

Cultures were declared negative after incubation for 5 days.

Antibiotic sensitivity pattern was assessed by the KirbyBauer disc diffusion method where discs containing antibiotics are placed on an agar plate on which bacteria were growing. If the bacteria are sensitive to the antibiotic, a zone of inhibition is seen around the disc.

Widal test was done by adding $0.5 \mathrm{ml}$ of Salmonella typhi O, Salmonella Typhi H, Salmonella paratyphi $\mathrm{A}(\mathrm{H})$ and Salmonella paratyphi $\mathrm{B}(\mathrm{H})$ antigens respectively in test tubes with diluted sera of 1:30.1:60, 1:120, 1:240 and 1:480 
concentration and also control of $0.5 \mathrm{ml}$ normal saline. After shaking the rack well, it was incubated at 37 degree centigrade overnight. Then with naked eye, the highest dilution in which there is evidence of agglutination is noted.

All the data were collected and then analysed using mainly descriptive statistics and Chi square analysis.

Of the 50 cases included in the study, majority i.e., 30 were 6-12 years of age. This is the school going age group and increased occurrence of enteric fever in this cohort implies the importance of proper sanitary facilities and stresses the need of preventing the disease by timely immunization programs.

Of the 20 cases, $<5$ years of age, 7 were $<1$ year. It may be related to poor hygiene or changing food patterns in recent years like early use of formula food products etc.

\section{Urban Versus Rural}

Out of 50 cases, $31(62 \%)$ were from rural area of West Bengal and the rest from urban area. The cause may be poor hygiene and improper sanitation.

Majority, 47 out of $50(94 \%)$ cases had fever lasting between 7-14 days, on admission. Only 3(6\%) cases, at the time of admission, had fever lasting for more than 2 weeks.

\section{Presenting Symptoms}

All the patients were febrile at the time of admission. Among the associated symptoms, gastrointestinal symptoms predominated in the majority. $50 \%$ had pain abdomen and $66 \%$ complained of constipation. Only 2 cases had a skin rash and dysuria at the time of admission.

Pain abdomen and constipation occurred almost equally in both age groups. Vomiting and diarrhoea were more common in 0-5 year's age cohort, while urinary symptoms though very rare was noticed in 6-12-year age group.

\section{Clinical Signs}

All the cases had hepatosplenomegaly. Pallor was present in $24(48 \%)$ cases, relative bradycardia in $14(28 \%)$ patients. Only 2 patients developed jaundice. Complications in the form of macrophage activating syndrome developed in 9 $(18 \%)$ cases.

Jaundice and coated tongue were common in 0-5 year's age group. Relative bradycardia was seen more often in 6-12 years age group. Other signs were more or less equal in both age groups.

\section{Laboratory Parameters \\ 1. Hb-}

In our study, majority had anaemia with Haemoglobin $<11$ gm/dl. Mean Hb was 10.27 with standard deviation of 1.7 ; minimum $\mathrm{Hb}$ was 4.6 and maximum was 13.3.

\section{Total Leucocyte Count}

Of the 50 cases normal leucocyte count was seen in $34(68 \%)$ cases. 12 patients had leukocytosis; the maximum count going up to 18300 . Only $4(8 \%)$ had leucopenia<4000/cumm; the minimum count being 2600.

Regarding the differential count, $20(40 \%)$ had normal neutrophil count but majority, 30(60\%) had neutropenia. Mean neutrophil count was 3757.76 with minimum neutrophil count being 1200 .
In our study, normal lymphocyte was seen in 38\% cases; $58 \%$ had lymphocytosis. Mean lymphocyte count was 3864.2.

\section{Platelet Count}

In our study, 41(82\%) had normal platelet count and thrombocytopenia was observed in $7(14 \%)$ cases. Thrombocytosis was rare, seen in only $2(4 \%)$ cases.

Erythrocyte Sedimentation Rate (ESR)-

Normal ESR was seen in 15 case while ESR $>20 \mathrm{~mm} / \mathrm{hr}$ was seen in $35(70 \%)$ cases.

\section{Liver Function Test (LFT)}

a. Only $2(4 \%)$ patients had raised bilirubin; maximum value being $4.3 \mathrm{mg} / \mathrm{dl}$.

b. Hemoglobinemia was noted in $7(14 \%)$ cases; the minimum level being $2.3 \mathrm{gm} / \mathrm{dl}$.

c. Transaminitis was seen in a good number of cases. SGOT (AST) was raised (mean value being 118.18 units/L) in 47(94\%) cases. SGPT (ALT) was raised in $29(58 \%)$ cases. The mean value was 80.04 units/L.

d. Altered coagulation profile $(\mathrm{INR}</=1.5)$ was seen in only $5(10 \%)$ cases.

Complications in the form of secondary HLH (Hemophagocytic lymphohistiocytosis) was seen in $9(18 \%)$ cases.

They had raised ferritin levels ( $>500$ microgram $/ L$ ). The maximum value of ferritin in such cases rose up to 4500 microgram/L. These patients also showed raised triglyceride (>265 mg/dl); the maximum being $650 \mathrm{mg} / \mathrm{dl}$.

Hypofibrinogenemia $(<150 \mathrm{mg} / \mathrm{dl})$ occurred in these cases, the minimum being $13 \mathrm{mg} / \mathrm{dl}$.

The Widal test was done 7 days or more after starting of fever, on all the 50 blood-culture positive cases. Widal test was positive in $26(52 \%)$ cases. T0 was positive 1:160 titre in $30 \%$ of cases.

In the above $2 \times 2$ contigency table, the Chi-square is 8.13 for association between the Widal test and Blood culture is statistically significant $(P<0.005)$ for $\alpha=0.05, d f=1$.

It can be inferred that that there is significant difference in the two tests. Out of 50 blood culture positive cases, Widal test ease positive in 26 and negative in 24 cases. Of the 40 blood-culture negative cases, only 9 showed positive Widal test. The Widal test has sensitivity $52 \%$, specificity $77.5 \%$; positive predictive value of $74.28 \%$ and negative predictive value of $56.36 \%$ when compared with blood culture result.

\section{Response to Therapy}

Of the 50 cases, 46 were antibiotic naïve and after admission parenteral ceftriaxone was started in them. 4 were already on oral antibiotics at the time of admission, which was thereafter continued.

46 cases were started therapy with ceftriaxone. Remaining 4 were already on treatment with oral azithromycin and cefixime at the time of admission. Of the 46 cases on ceftriaxone, 36 cases out of 50 (72\%) responded within 3-5 days of initiation of antibiotic therapy. 10 cases (20\%) did not show defervescence even after 1 week of ceftriaxone injection. 
They however responded to other antibiotics like meropenem ( 9 cases), 1 of each responded to co-amoxiclav, cefixime and ofloxacin. 2 responded to azithromycin.

The mean period of defervescence was 4.78 days. Minimum and maximum time taken to achieve defervescence was 2 and 13 days respectively.

Of the 10 cases which failed to show response to ceftriaxone, 9 achieved remission with meropenem and became afebrile 2-4 days after starting therapy with meropenem. Only 1 case continued to have fever even after 1 week of meropenem, and eventually responded to parenteral ofloxacin.

\section{DISCUSSION}

The above study was conducted as a retrospective observational study among the inpatient of VIMS, (Department of Paediatrics) whose blood culture was positive for salmonella typhi. This study spanned over a period of 18 months.

Of the cases included in the study, 20 cases (40\%) fell in $0-5$ years age group and 30 cases $(60 \%)$ fell in $6-12$ years age group. Of them 7 cases (14\%) were less than 1 year. Walia et al(1) reported that $21.7 \%$ were children aged under 5 years and $6.1 \%$ were under 2 years. Yaramis et al(2) reported that $17 \%$ of patients were children under 5 years and that school children were most affected.

In our study, 26 out of the 50 cases were males and amounted to $52 \%$ and 24 cases were females and amounted to $48 \%$. Such male predominance was also noted in the studies by Garg et $\mathrm{al}^{(3)}$ and Raghuram et al.(4)The male preponderance in our study was noted in the $<5$ years age group.

Like all other studies, fever in this study was the universal presenting feature. In 30 cases (60\%) fever at the time of presentation, had a duration of $<7$ days. Only 3 cases $(6 \%)$ it was $>2$ weeks duration.

Pain abdomen was seen in $66 \%$ cases, which was much higher than reported by R K Arora et al(5) and Madhavan et al.(6)

Diarrhoea was seen in $12 \%$ cases, which was similar to that reported by R K Arora et al.(5)

Among gastro intestinal symptoms, vomiting and diarrhoea were common $<5$ years age, while urinary symptoms like dysuria was commoner $>6$ years of age. Pain abdomen and constipation occurred equally in both age groups.

Among the clinical findings, relative bradycardia was seen in $28 \%$ cases which is less than reported by Madhavan et al(6) who cited it to be as high as $44.7 \%$.

Pallor was seen in $48 \%$ cases, which is similar to R K Arora et al. In cases of severe fall in haemoglobin one should be alert of a massive GI bleed or other complications like secondary HLH.

Jaundice was seen in only $4 \%$ cases, which is similar to that reported by Chandrasekhar et al.(7)

Hepatosplenomegaly was a consistent feature of this study, which is similar to that reported by R K Arora et al.

Sinha et al(8) reported that enteric fever may present in a dramatic form, 5 years of age who have higher percentage of complications and more frequent hospitalization.

In our study $68 \%$ had normal leucocyte counts and only $8 \%$ had leucopenia; leukocytosis was seen in $24 \%$ cases.
Almost similar incidence of leucopenia was also reported by $\mathrm{R} K$ Arora et al (10.7\%) and Chungathu et al (8\%).

$58 \%$ cases in our study was reported to have relative lymphocytosis. A similar picture was also observed by Chungathu et al(9) who cited lymphocytosis to the tune of $44 \%$.

Thrombocytosis was seen in $14 \%$ cases; Madhavan et al reported it to be $27.6 \%$.

Transaminitis -SGOT \& SGPT were raised in $94 \%$ and $58 \%$ cases respectively. This observation was however higher compared to Chandrasekhar et al (8\%) and Madhavan et al.

Widal test was compared with blood culture in the initial 90 case records.

Chi-square 8.13 for the association between the Widal test and blood culture test is significant $(\mathrm{p}<0.005)$.

Thus, it can be conferred that there is a significant difference in the two tests.

Out of the 50 blood-culture positive cases only 26 were Widal positive. Of the 40 blood-culture negative cases, 9 were Widal positive. Widal test has a sensitivity of $52 \%$ and specificity of $77.5 \%$. When compared to conventional blood culture, Widal test had a positive predictive value of $74.28 \%$ and a negative predictive value of $56.36 \%$.

Of the 50 blood-culture positive cases, Widal was positive in 26 case, T0 titre $>1: 160$ was seen in $30 \%$ cases. This was in contrast to the observation made by Chandrasekhar et al (T0>1:160 seen in $92.3 \%$ cases).

Of the 50 cases, 46 were antibiotic naïve at the time of admission. Parenteral ceftriaxone was started in them. 36 responded to it. 10 continued to have fever even after 7 days of ceftriaxone; guided by the culture sensitivity reports, meropenem was initiated in these non-responsive cases. 9 became afebrile, one needed parenteral ofloxacin as fever did not remit even after 1 week of meropenem.

\section{CONCLUSIONS}

Out of the 50 blood-culture positive salmonella typhi enteric fever, $60 \%$ belonged to the school going age group of 6-12 years. Males outnumbered females $<5$ years age group. The consistent features were fever and hepatosplenomegaly. Majority had normal leucocyte count with relative lymphocytosis. Widal test though a simple, cheap and readily available test, it's reliability a diagnostic tool is questionable because not all blood culture positive patients showed a positive Widal test. Majority of the cases were resistant to quinolones and aminoglycosides and all were resistant to nalidixic acid. So, they cannot be recommended for therapy. Majority, 96\% were sensitive to ceftriaxone but clinical response was seen in $78.3 \%$ cases. $21.7 \%$ did not respond/deteriorated further even after 1 week of treatment. Meropenem response was seen in $90 \%$ cases; only $10 \%$ showed resistance and later responded to ofloxacin. Of the 50 cases, 9 developed complications like secondary HLH even while on therapy with ceftriaxone. They later responded to meropenem. However, majority responded to ceftriaxone. In spite of this, ceftriaxone still remains the drug of choice in uncomplicated enteric fever. Oral Azithromycin and Cefixime also showed a good response and a high sensitivity pattern was noted with both of them. 


\section{REFERENCES}

[1] Walia M, Gaind R, Mehta R, et al. Current perspectives of enteric fever: a hospital-based study from India. Ann Trop Paediatric 2005;25(3):161-74.

[2] Yaramis A, Yildirim I, Katar S, et al. Clinical and laboratory presentation of typhoid fever. International Pediatrics 2001;16(4):227-31.

[3] Garg RA, Krashak R. Typhoid fever before 2 years of age. Indian Pediatrics 1993;30(6):805-7.

[4] Raghuraman TS, Krishnamurthy L, Menon PK, et al. Clinical profile and therapy in enteric fever. Indian Pediatrics 1993;30.

[5] Arora RK, Gupta A, Joshi NM, et al. Multidrug resistant typhoid fever: study of an outbreak in Calcutta. Indian Pediatrics 1992;29(1):61-6.
[6] Madhavan K, Priyadarshini V, Sivaprakash V, et al. A study on clinical, laboratory profile and drug sensitivity pattern in Salmonella positive patients. Int J Sci Stud 2017;5(1):35-9.

[7] Chandrashekar, AnilKumar YC, Kirandeep S, et al. Study of clinical and laboratory profile of enteric fever in pediatric age group. International Journal of Basic and Applied Medical Sciences 2013;3(3):16-23. http://www.cbtech.org/jms.htm

[8] Sinha A, Sazawal S, Kumar R, et al. Typhoid fever in children aged less than 5 years. The Lancet 1999;354(9180):734-7.

[9] http://repositorytnmgrmu.ac.in/6940/1/200701213riyas_chungathu. 